\title{
Light-Cone Bootstrap of Higher Point Functions and Wilson Loop Duality
}

\author{
Carlos Bercini $\odot,{ }^{1}$ Vasco Gonçalves, ${ }^{1,2}$ and Pedro Vieira ${ }^{3,1}$ \\ ${ }^{1}$ ICTP South American Institute for Fundamental Research, IFT-UNESP, So Paulo 01140-070, Brazil \\ ${ }^{2}$ Centro de Física do Porto e Departamento de Física e Astronomia, \\ Faculdade de Ciências da Universidade do Porto, Porto 4169-007, Portugal \\ ${ }^{3}$ Perimeter Institute for Theoretical Physics, Waterloo, Ontario N2L 2Y5, Canada
}

(Received 17 September 2020; revised 14 December 2020; accepted 25 February 2021; published 26 March 2021)

\begin{abstract}
We initiate an exploration of the conformal bootstrap for $n>4$ point correlation functions. Here we bootstrap correlation functions of the lightest scalar gauge invariant operators in planar non-Abelian conformal gauge theories as their locations approach the cusps of a null polygon. For that we consider consistency of the OPE in the so-called snowflake channel with respect to cyclicity transformations which leave the null configuration invariant. For general non-Abelian gauge theories this allows us to strongly constrain the OPE structure constants of up to three large spin $J_{j}$ operators (and large polarization quantum number $l_{j}$ ) to all loop orders. In $\mathcal{N}=4$ we fix them completely through the duality to null polygonal Wilson loops and the recent origin limit of the hexagon explored by Basso, Dixon, and Papathanasiou.
\end{abstract}

DOI: $10.1103 /$ PhysRevLett.126.121603

Introduction.-The numerical conformal bootstrap [1] is by now a well-established physics tool, often leading to the best determination of critical exponent relevant for realworld experiments. The so-called analytical conformal bootstrap explores a corner of the bootstrap-usually the very Lorentzian domain - to derive universal analytical results for general conformal field theories or general conformal gauge theories.

A beautiful example is the work of Alday and Bissi [2]. They found the planar $\mathcal{N}=4$ structure constant between the lightest single trace scalars of the theory and the leading twist single trace operators with large spin $J$ as

$$
\hat{C}^{\circ \circ \bullet} \simeq \mathcal{N}^{\circ \circ} \Gamma\left(1-\frac{1}{2} \gamma\right) e^{-\frac{1}{2}(f \ln 2+g) \ln J}
$$

Here and below, the hat in $\hat{C}$ stands for the all loop structure constant normalized by the tree level result and $\mathcal{N}^{\circ{ }^{\circ}}$ is a coupling dependent (but spin independent) normalization constant (which bootstrap arguments will always be insensitive to), [3]. The functions $f$ and $g$ are, respectively, the cusp and collinear anomalous dimensions. They show up in the anomalous dimension $\gamma$ of the leading twist operators at large spin $J$, which exhibits logarithmic scaling [4] and behave as

Published by the American Physical Society under the terms of the Creative Commons Attribution 4.0 International license. Further distribution of this work must maintain attribution to the author(s) and the published article's title, journal citation, and DOI. Funded by SCOAP ${ }^{3}$.

$$
\gamma \simeq f(\lambda) \ln (J)+g(\lambda)
$$

in any gauge theory. Although extensively checked in $\mathcal{N}=4$, where we have abundant perturbative data, the derivation is really a bootstrap one and as such the prediction (1) is actually expected to hold for any large $N$ conformal gauge theory.

In principle, the study of all 4 point correlation functions contains information about all the CFT data. In practice most bootstrap studies focus on a single correlator or on a coupled system involving a few 4 point correlators. Here we propose to use higher point functions as well. Since they contain, in their OPE, infinitely many 4 point correlation functions, we can expect that a great deal of information can be extracted from them.

We will obtain several new analytic results akin to Eq. (1) but involving several spinning operators, namely, Eqs. (16) and (20). Our results will be valid for general conformal gauge theories in the planar limit but our testing ground will once again be planar $\mathcal{N}=4$ SYM. Better understanding the various dualities relating scalar correlation functions, null polygonal Wilson loops (WL), gluon scattering amplitudes and large spin correlation functions [5-10] in this gauge theory is another motivation for these bootstrap exercises. Correlation functions correspond to AdS closed string scattering and are nicely built out of integrable hexagons [11] while null WL can be depicted as open string partition functions and can be constructed out of integrable pentagons [12]. We hope these explorations will lead to a unifying description of both.

Kinematics.-The OPE of two operators on the light cone was written around 50 years ago [13]: 


$$
\mathcal{O}\left(x_{1}\right) \mathcal{O}\left(x_{2}\right) \sim \frac{C\left(2 x_{12} \cdot \partial_{\epsilon}\right)^{J}}{\left(x_{12}^{2}\right)^{\left\{\left[2 \Delta_{\phi}-(\Delta-J)\right] / 2\right\}}{ }_{1}} F_{1}\left(\frac{\Delta+J}{2}, \Delta+J, x_{21} \cdot \partial_{x_{1}}\right) \mathcal{O}_{\Delta, J}\left(x_{1}, \epsilon\right),
$$

where $C$ is the OPE coefficient. Here $\epsilon$ is a polarization vector and we should only keep the leading terms in $x_{12}^{2}$. This operator identity can be used to derive integral representations for conformal blocks by applying it once, twice, or three times in a 4,5 , and 6 point correlator, respectively. After these multiple OPEs end up with the conformal block as the action of a few ${ }_{1} F_{1}$ operators on a spinning 3 point function which in turn is completely fixed by conformal symmetry [14]:

$$
\left\langle\mathcal{O}\left(x_{1}, \epsilon_{1}\right) \ldots \mathcal{O}\left(x_{3}, \epsilon_{3}\right)\right\rangle=\sum_{l_{i j}} C_{J_{1} J_{2} J_{3}}^{l_{12} l_{13} l_{23}} \frac{V_{1,23}^{J_{1}-l_{12}-l_{13}} V_{2,31}^{J_{2}-l_{12}-l_{23}} V_{3,12}^{J_{3}-l_{13}-l_{23}} H_{12}^{l_{12}} H_{31}^{l_{13} 3} H_{23}^{l_{23}}}{\left(x_{12}^{2}\right)^{\left[\left(\tau_{1}+\tau_{2}-\tau_{3}\right) / 2\right]}\left(x_{13}^{2}\right)^{\left[\left(\tau_{1}+\tau_{3}-\tau_{2}\right) / 2\right]}\left(x_{23}^{2}\right)^{\left[\left(\tau_{2}+\tau_{3}-\tau_{1}\right) / 2\right]}},
$$

where $\epsilon_{i}$ are the polarization vectors, $\tau_{i}=\Delta_{i}+J_{i}$ are the conformal spin of the operators, and the $V$ and $H$ tensors are recalled in the Supplemental Material [15]. The integers $l_{i j}$ parametrize the various possible tensor structures where the polarizations vectors show up and we simply call them polarizations. Their range is such that all exponents in Eq. (4) are non-negative. (The 5 point block only depends on two spins $J_{1}, J_{2}$ and a single nonzero polarization $l \equiv l_{12}$.) Finally, we use the standard integral representation for each ${ }_{1} F_{1}$ to obtain a useful expression for the conformal blocks

$$
F_{n}=\operatorname{kin}_{n} \int_{0}^{1}\left[d y_{1}\right] \ldots\left[d y_{n-3}\right] \text { int }_{n},
$$

where the explicit expressions for the kinematical prefactors, integrands, and measure are summarized in the Supplemental Material [15].

Correlation functions of 5 and 6 points depend on 5 and 9 independent cross ratios, respectively. For the $5 \mathrm{pt}$ function, a convenient choice are the cross ratios [18]

$$
u_{1} \equiv \frac{x_{12}^{2} x_{35}^{2}}{x_{13}^{2} x_{25}^{2}}, \quad x_{i j}^{2} \equiv\left(x_{i}-x_{j}\right)^{2}
$$

while all other four cross ratios are trivially obtained by simply shifting the indices here,

$$
\left.u_{i} \equiv u_{i-1}\right|_{x_{i} \rightarrow x_{i+1}}
$$

where $i=2,3,4,5$; see Fig. 1 . These cross ratios contain a single nearest neighbor distance $\left[x_{12}\right.$ in Eq. (6)]. The lightcone OPE in the channel 12 and 34 channel projects into leading twist contributions and can be achieved by taking $u_{1}, u_{3} \rightarrow 0$; see Fig. 2. For $x_{15}^{2}, x_{45}^{2} \rightarrow 0$ ( or $u_{4}, u_{5} \rightarrow 0$ ) we are further dominated by large spin operators. Then the leading twist blocks simplify dramatically into a simple product of Bessel functions

$$
F_{5} \simeq \frac{\left(1-u_{2}\right)^{l}}{\pi} \prod_{i=1}^{2} \frac{2^{2 J_{i}+\gamma_{i}} J_{i}^{\frac{1}{2}}}{u_{1-i}^{-\left[\left(2 l+\gamma_{i+1}\right) / 4\right]}} K_{l+\left[\left(\gamma_{i+1}\right) / 2\right]}\left(2 J_{i} \sqrt{u_{1-i}}\right) .
$$

At this point, the polarization $l$ is still finite; in the full null pentagon limit where all $x_{i, i+1}^{2} \rightarrow 0$ (i.e., all $u_{i} \rightarrow 0$ ) we project into the limit of large polarization further simplifying these Bessel functions into simple exponentials, see (A9) in the Supplemental Material [15].

For 6 point functions we define 6 cross ratios $u_{j}$ through Eqs. (6) and (7), where $i$ now runs up to 6 in the second relation. The remaining 3 cross ratios $\left(U_{1}, U_{2}, U_{3}\right)$ which parametrize the 6 point functions are

$$
U_{1} \equiv \frac{x_{13}^{2} x_{46}^{2}}{x_{14}^{2} x_{36}^{2}}, \quad U_{2}=\left.U_{1}\right|_{x_{i} \rightarrow x_{i+1}}, \quad U_{3}=\left.U_{2}\right|_{x_{i} \rightarrow x_{i+1}} ;
$$

see Fig. 1. Again, we can explore a null hexagon limit by taking all consecutive points to become null separated. First we take 12,34 , and 56 to become null by taking $u_{1}, u_{3}, u_{5}$ to zero; this projects into leading twist operators (with finite spins $J_{1}, J_{2}, J_{3}$ ) in these three OPE channels. Next we take

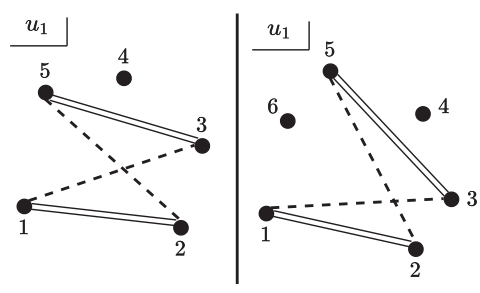

(a)

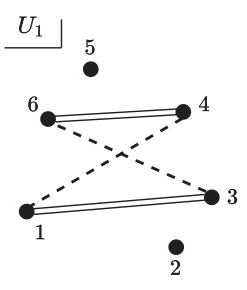

(b)
FIG. 1. (a) We pick the five independent cross ratios for the $5 \mathrm{pt}$ function, as $u_{1}$ represented here plus its four cyclic images. (b) For the $6 \mathrm{pt}$ function, we choose the nine independent cross ratios as $u_{1}\left(U_{1}\right)$ represented here plus its five (two) cyclic images. 

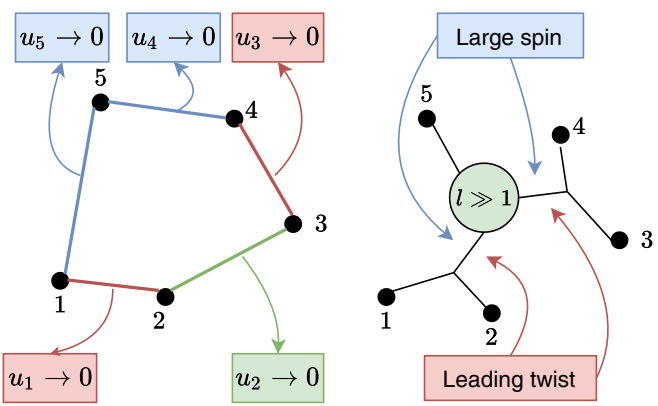

FIG. 2. Each time we take consecutive points $x_{i}$ and $x_{i+1}$ to become null separated, we have $u_{i} \rightarrow 0$. We can thus construct a full null pentagon sequentially: $u_{1,3} \rightarrow 0$ projects into leading twist; $u_{4,5} \rightarrow 0$ projects into large spin; finally $u_{2} \rightarrow 0$ is controlled by structure constants with large $l$.

23,45 , and 61 to become null by taking $u_{2}, u_{4}, u_{6}$ to zero; this projects into large spins $J_{i} \gg 1$; see Fig. 3. We consider a corner of this limit where we also take 24 , 46, and 62 to become null; see Fig. 3. If they become null with the same rate the conformal blocks simplify considerably in this limit and are dominated by large spin $J_{i}$,

$$
F_{6} \simeq \prod_{i=1}^{3} \frac{2^{2 J_{i}+\gamma_{i}} J_{i}^{\frac{1}{2}} K_{l_{i-1}-l_{i}+\left[\left(\gamma_{i}-\gamma_{i-1}\right) / 2\right]}\left(2 J_{i+1} \sqrt{U_{2-i}}\right)}{U_{2-i}^{-\left[\left(\gamma_{i}-\gamma_{i-1}+2 l_{i}-2 l_{i-1}\right) / 4\right]}\left(U_{2-i}-u_{2 i}\right)^{-l_{i-1}}},
$$

where $l_{1}=l_{23}, l_{2}=l_{13}, l_{3}=l_{12}$ are kept finite and where all indices are understood in the cyclic sense with respect to their range, so that $U_{-2}=U_{3-2}=U_{1}$ and so on. We denote this limit - where all cross ratios vanish with the same rate- as the generalized origin limit. Now, if we take the diagonals to approach the null limit faster than the perimeter, then $U_{i} \ll u_{i}$ and we are dominated by small polarizations, $l_{i j}=0$. We will not explore this interesting limit here. If, on the contrary, we take the perimeter to become null faster than the inner diagonals then $u_{i} \ll U_{i}$ and we are dominated by large polarizations. [Then we can simplify the blocks into exponentials as in the 5 pt case, see

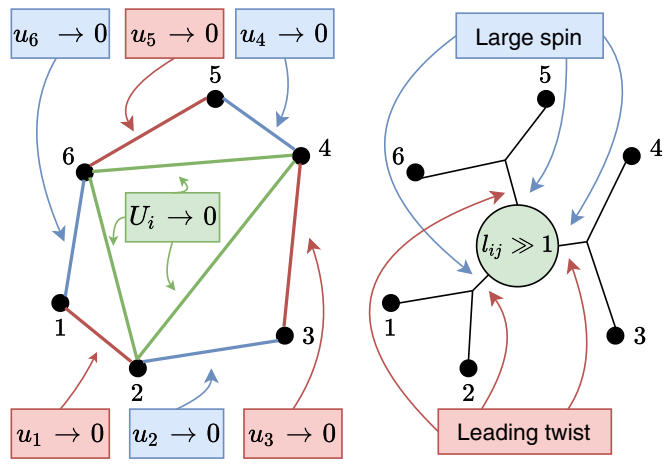

FIG. 3. Consecutive $6 \mathrm{pt}$ limits: $u_{1,3,5} \rightarrow 0$ projects into leading twist; $u_{2,4,6} \rightarrow 0$ projects into large spin; finally $U_{i} \rightarrow 0$ is controlled by structure constants with large $l_{i j}$.
(A9) in the Supplemental Material [15].] This limit preserves cyclicity and is the most natural one from a WL duality point of view as we are first constructing the polygon boundary and only then considering the simplifying limit $U_{i} \rightarrow 0$.

There is another set of cross ratios $v_{i}\left(u_{i, i+1}\right.$ in Ref. [18]),

$$
v_{i} \equiv \frac{x_{i-1, i}^{2} x_{i+1, i+2}^{2}}{x_{i-1, i+1}^{2} x_{i, i+2}^{2}}
$$

which are completely local and valid for any polygon but contain two vanishing distances (inconvenient when taking a null limit at a time). We will use the single null distance cross ratios $u_{i}$ to bootstrap the results which we present using the local cross ratios $v_{i}$.

Bootstrap.-Cyclic correlators: We consider $n$-pt functions of the lightest single trace operator. Since we are after a full null limit where the various external points approach the vertices of a null polygon, it is convenient to make cyclicity manifest by stripping out a cyclic space-time dependent factor. For example, in planar $\mathcal{N}=4 \mathrm{SYM}$ we consider $n$-point functions of $20^{\prime}$ operators $\mathcal{O}_{j} \propto$ $\operatorname{tr}\left[\left(y_{j}\right)_{A} \phi^{A}\left(x_{j}\right)\right]^{2}$ and write

$$
\left\langle\prod_{i=1}^{n} \mathcal{O}_{j}\right\rangle=\prod_{i=1}^{n} \frac{y_{i} \cdot y_{i+1}}{\left(x_{i}-x_{i+1}\right)^{2}} \times G_{n} .
$$

Then $G_{n}$ is a cyclic function of the conformal cross ratios alone [19]. We pick the normalization such that $G_{n}=$ $1+\mathcal{O}(\lambda)$ at tree level. The simplest cyclic correlator is $G_{4}$ which in the null square limit was bootstrapped in Ref. [2]. Cyclicity $(u \leftrightarrow v)$ combined with the large spin limit described in Eq. (1) fixes the correlator to be

$$
\begin{aligned}
G_{4} & \simeq e^{\Sigma_{i=1}^{4} \ln v_{i}\left[(g / 4)-(f / 16) \ln v_{i+1}\right]} \\
& \times\left(\mathcal{N}^{\circ \bullet \bullet}\right)^{2} \prod_{i=1}^{4} e^{-(4 / f)\left(\partial^{2} / \partial \ln v_{i} \partial \ln v_{i+1}\right)} \prod_{i=1}^{4} \Gamma\left(1-\frac{g}{2}+\frac{f}{4} \ln v_{i}\right),
\end{aligned}
$$

where the $v_{j}$ relate to the conventional $4 \mathrm{pt}$ function cross ratios as $v_{1}=v_{3}=v$ and $v_{2}=v_{4}=u$ [20].

5 pt function: The bootstrap of the cyclic $5 \mathrm{pt}$ function $G_{5}$ in the null polygon limit follows closely the 4 pt function bootstrap of [2]. We plug a perturbative ansatz

$$
\hat{P}^{\circ \cdots \bullet} \simeq \sum_{k=0}^{\infty} \lambda^{k} \sum_{i_{k}} c_{k, i_{1}, i_{2}, i_{3}} \times \ln ^{i_{1}} J_{1} \ln ^{i_{2}} J_{2} \ln ^{i_{3}} l
$$

for the relevant combination of structure constants

$$
\hat{P}^{\circ \bullet \bullet}=\hat{C}^{\circ \bullet \bullet}\left(J_{1}, J_{2}, l\right) \hat{C}^{\circ \circ \bullet}\left(J_{1}\right) \hat{C}^{\circ \circ \bullet}\left(J_{2}\right),
$$

arising in the OPE decomposition of the $5 \mathrm{pt}$ function 
$G_{5} \simeq \int_{0}^{\infty} \frac{1}{4} \hat{P}^{\circ \cdot \bullet} u_{1}^{1+\left\{\left[\gamma\left(J_{1}\right)\right] / 2\right\}} u_{3}^{1+\left\{\left[\gamma\left(J_{2}\right)\right] / 2\right\}} \breve{F}_{5} d j_{1} d j_{2} d l$,

and compute this object to any desired order in perturbation theory [21]. The integrals involved are very similar to those arising in the $4 \mathrm{pt}$ function case of Ref. [22], see Supplemental Material [15].

In this way we obtain $G_{5}$ in the polygon limit expressed in terms of the unfixed constants $c_{k, i_{1}, i_{2}, i_{3}}$ in the structure constants (13) and of the collinear and cusp anomalous dimensions appearing in Eq. (2). Now, because we picked a particular OPE channel for the decomposition [see Fig. 2(b)] the whence obtained result will not be automatically cyclic. Imposing cyclicity (i.e., invariance under $u_{i} \rightarrow u_{i+1}$ ) will thus strongly constrain the unfixed constants $c_{k, i_{1}, i_{2}, i_{3}}$, resulting in

$$
\hat{C}^{\circ \bullet \bullet} \simeq \mathcal{N}^{\circ \bullet \bullet} e^{-\{[f(\lambda)] / 4\}\left[\ln ^{2} l+\ln 4 \ln \left(J_{1} J_{2}\right)\right]-\{[g(\lambda)] / 2\} \ln l} .
$$

This all loop result for the large spin and large polarization behavior of the OPE coefficients involving two spinning operators is the counterpart of Eq. (1) with a single spinning operator [23]. Having completely constrained the structure constants in the OPE decomposition, we can then write down the correlator itself as

$$
G_{5} \simeq e^{(-f / 16)\left(\sum_{i=1}^{5} \ln v_{i}\right)^{2}+\Sigma_{i=1}^{5} \ln v_{i}\left[(g / 4)+(f / 4) \ln v_{i+2}\right]} \mathcal{N}^{\circ \bullet}\left(\mathcal{N}^{\circ \bullet \bullet}\right)^{2} \prod_{i=1}^{5} e^{-(4 / f)\left(\partial^{2} / \partial \ln v_{i} \partial \ln v_{i+1}\right)} \prod_{i=1}^{5} \Gamma\left(1-\frac{g}{2}+\frac{f}{4} \ln v_{i}\right)
$$

We interpret it physically in the next section. We compared our results with perturbative one-loop results in the literature. In the limit of large spin and polarization, the correlation function (17) agrees with the one-loop computations of Ref. [26] and the structure constant (16) agrees with the perturbative computations of Ref. [27].

6 pt function: For the $6 \mathrm{pt}$ function we focus on the origin limit, where we plug the ansatz

$$
\hat{P}^{\cdots \cdots} \simeq \sum_{k=0}^{\infty} \lambda^{k} \sum_{i_{k}} c_{k, i_{1}, \ldots, i_{6}} \prod_{n=1}^{3} \ln ^{i_{n}} J_{n} \ln ^{i_{n+3}} l_{4-n}
$$

$\left(i_{1}+i_{2}+i_{3} \leq k, i_{1}+\cdots+i_{6} \leq 2 k\right)$ for the relevant combination $\quad \hat{P}^{\cdots *}=\hat{C}^{\cdots \cdot \bullet}\left(J_{1}, J_{2}, J_{3}, l_{1}, l_{2}, l_{3}\right) \prod_{j=1}^{3} \hat{C}^{\circ * \bullet}\left(J_{j}\right)$ appearing in the OPE decomposition

$$
\begin{aligned}
G_{6} \simeq & \int_{0}^{\infty} \frac{1}{8} \hat{P}^{\cdots \cdots} u_{1}^{1+\left\{\left[\gamma\left(J_{1}\right)\right] / 2\right\}} u_{3}^{1+\left\{\left[\gamma\left(J_{2}\right)\right] / 2\right\}} u_{5}^{1+\left\{\left[\gamma\left(J_{3}\right)\right] / 2\right\}} \\
& \times \check{F}_{6} \prod_{i=1}^{3} d j_{i} d l_{i}
\end{aligned}
$$

where the block is given by (A10) and we impose cyclicity (i.e., invariance under $u_{i} \rightarrow u_{i+1}$ and $\left.U_{i} \rightarrow U_{i+1}\right)$. At loop order $k$, we find that all power of logs in Eq. (18) from degree $k$ up to $2 k$ are fixed, while the remaining constants are such that only the three variables $\mathbb{L}_{j} \equiv 2 \log \left(J_{j}\right)-$ $\log \left(l_{j-1}\right)-\log \left(l_{j-2}\right)$ show up. That is, cyclicity fixes the large spin and large polarization structure constant to be

$$
\begin{aligned}
\hat{C}^{\cdots \bullet} \simeq & \mathcal{N} \cdots e^{\Sigma_{i=1}^{3}\{[f(\lambda)] / 2\} \ln J_{i} \ln \left[\left(J_{i} l_{i}\right) /\left(2 l_{i+1} l_{i+2}\right)\right]} \\
& \times \exp \left(-\frac{f(\lambda)}{8} \sum_{i=1}^{3}\left(\mathbb{L}_{j}\right)^{2}+\sum_{k \geq 1} \lambda^{k} P_{k}\left(\mathbb{L}_{1}, \mathbb{L}_{2}, \mathbb{L}_{3}\right)\right),
\end{aligned}
$$

which translates into the associated $6 \mathrm{pt}$ function in the origin limit as

$$
\begin{aligned}
\hat{G}_{6} \simeq & e^{\Sigma_{i=1}^{6}(f / 16) \ln v_{i} \ln v_{i+3}-(f / 8) \ln v_{i} \ln v_{i+1}+(g / 4) \ln v_{i}} \mathcal{N}^{\cdots} \cdot\left(\mathcal{N}^{\circ \bullet}\right)^{3} \prod_{i=1}^{6} e^{-(4 / f)\left(\partial^{2} / \partial \ln v_{i} \partial \ln v_{i+1}\right)} \prod_{i=1}^{6} \Gamma\left(1-\frac{g}{2}+\frac{f}{4} \ln v_{i}\right) \\
& \times e^{-\{[f(\lambda)] / 8\} \Sigma_{i=1}^{3}\left(\log U_{j}\right)^{2}+\Sigma_{k \geq 1} \lambda^{k} P_{k}\left(\log U_{1}, \log U_{2}, \log U_{3}\right),}
\end{aligned}
$$

where $P_{k}$ are undetermined totally symmetric polynomials of three variables of degree $k$. In the original ansatz (18) we had polynomials of twice as many variables and twice the degree; see Table I.

WL relation.-In the null polygon limit all correlation functions bootstrapped here can be compactly written as [18]

$$
G_{n}=(\text { Sudakov })_{n} \times(\text { J Recoil })_{n} \times \mathbb{W}_{n} .
$$

The first factor contains the leading divergences of the correlator developed as it approaches the null polygon limit. These physical divergences are identified with UV cusp divergences of the dual null polygonal WL [18] or IR divergences of the dual gluon scattering amplitudes [28] (in $\mathcal{N}=4$ SYM). This Sudakov factor is given in magenta in first lines in our expressions (12), (17), and (21) and precisely matches with the prediction of Ref. [18]. 
TABLE I. Number of free parameters in $C^{\cdots \cdot *}$ at large $J_{i}, l_{i j}$.

\begin{tabular}{lcc}
\hline \hline Loop order & Perturbative ansatz (18) & After cyclicity \\
\hline 1 & 8 & 2 \\
2 & 53 & 4 \\
3 & 243 & 8 \\
4 & 708 & 13 \\
5 & 1862 & 20 \\
\hline \hline
\end{tabular}

The second factor should be identified with a subtle backreaction effect: as the correlation function insertions become null separated, fast particles will propagate between consecutive insertions thus dynamically generating a WL. However, these charged particles interact with each other and get pulled towards each other producing a recoil effect which needs to be taken into account. This $J$ recoil factor is written in blue in the second line in our expressions (12), (17), and (21). It was given in Ref. [18] at two loops and derived to all loops for $n=4$ by Alday and Bissi [2]. Here we derived its all loop expression for $n=5$ and $n=6$; our results agree with the two loop predictions of Ref. [18]. Note that the $J$ recoil factor and the Sudakov factor only depend on the local cross ratios $v_{i}$, which go to zero in the null polygon limit. The $n>6$ extrapolation of our findings seems obvious as well.

Finally, we have the renormalized conformal WL factor $\mathbb{W}_{n}$, which is finite as we approach the null limit. It is equal to 1 for $n=4,5$ since there are no cross ratios to be constructed out of null squares or pentagons while for $n=6$ it only depends on the three finite cross ratios $U_{i}$ and is directly related to the hexagon null WL or 6 point MHV gluon scattering amplitude in planar $\mathcal{N}=4$ SYM. (In the notation of Ref. [29], we expect $\mathbb{W}_{6}=e^{2 \mathcal{E}}$.) This WL factor is given (in green) in the third line in Eq. (21) in the limit where all $U_{i}$ are taken to be very small. Now, in the recent work of Ref. [29] it was observed that in this limit the WL expectation value in planar $\mathcal{N}=4$ SYM exponentiates into a simple quadratic form in $\ln \left(U_{j}\right)$ with coefficients given in terms of two functions $\Gamma_{\text {oct }}$ and $\Gamma_{\text {hex }}$, which are explicitly given [30]. We conclude that our symmetric polynomials are simply quadratic and read

$$
\begin{aligned}
\sum_{l} \lambda^{l} P_{l}= & 2 C_{0}+\frac{3 \Gamma_{\text {cusp }}-\Gamma_{\text {oct }}-2 \Gamma_{\text {hex }}}{12} \sum_{j=1}^{3} \ln ^{2}\left(U_{j}\right) \\
& -\frac{\Gamma_{\text {oct }}-\Gamma_{\text {hex }}}{12} \sum_{j \neq k} \ln U_{k} \ln U_{j},
\end{aligned}
$$

thus fixing the large spin, large polarization structure constants $C^{\cdots \cdots}$ to all loop orders in this gauge theory. (Here $\Gamma_{\text {cusp }}=f / 2$ is the fundamental cusp anomalous dimension.)

Conclusion.-We explored full light-cone OPE limits in 5 point and 6 point functions in large $N$ gauge theories. In these limits, the operators approach the vertices of null polygons and the correlators are dominated by the exchange of single trace leading twist operators with large spin.

Five point correlation functions (and corresponding large spin structure constants $C^{\bullet \bullet}$ ) can be fully determined to all loops in this limit [33]. Six point functions around the origin limit (and corresponding structure constants $C^{\text {*.* }}$ involving large spins and polarizations) are strongly constrained-see Table I-in generic gauge theories and totally fixed in $\mathcal{N}=4$ super Yang-Mills through the relation to the origin limit of null polygonal WLs [29].

We believe we are only scratching the surface of a very promising precise connection between (integrated) correlation functions and (integrated) WL. It would be very interesting, for instance, to consider a systematic expansion of correlation functions around the large spin limit and large polarizations considered here and see how this related to the expansion around the origin in the WL side. And of course, there is a plethora of physical WL-amplitudes limits one could consider and translate for the correlation function side.

We explored the conformal bootstrap for 6 point functions by OPE expanding the external operators in pairs and then considering the resulting spinning 3 point function. This is the so-called snowflake decomposition [35]. Another OPE decomposition is the so-called comb decomposition where OPEs are taken in a sequential way. Conformal block in this limit can be found in Refs. [35-37]. Could further constraints from the comb decomposition fix the correlator in the origin limit or perhaps shed light on the quadratic truncation in $\log U_{i}$ found in Ref. [29]? The comb decomposition reminds us of the POPE decomposition [12], where each pentagon is concatenated after another; it might be the natural decomposition to recover the WL collinear limit.

We hope these games will also pave the way towards a unified integrability description of open and closed strings in AdS/CFT since they relate closed string scattering (correlation functions) to open strings partition functions (WL). Recovering the all loop large spin 3 point functions results derived here through the hexagon formalism [11] should be very illuminating in this regard. Of course, to make progress we will also need more perturbative data. In the open string pentagon OPE program [12], for instance, such perturbative data was key in validating several integrability based conjectures, see, e.g., Ref. [38]. Hexagonal and heptagonal WLs have been computed to mind-blowing perturbative orders; see Ref. [39] for a recent review. The same cannot be said about higher point correlation functions. Apart from some important $n=5$ two loop integrand results in Ref. [28] and the one loop integrated correlators in Ref. [26], virtually nothing is known about $n>4$ correlators. The 2-loop integrated $6 \mathrm{pt}$ function, for instance, would be extremely useful for 
checking any integrability-bootstrap based formulation since at 1-loop things are often misleadingly simple.

It would be interesting to study the case where the OPE is dominated in some channels by a finite number of exchanged operators. This would be a generalization of Refs. [40,41] to higher a number of points.

The exploration of null pentagons and hexagons provides us with a plethora of exact results which seem hardly attainable through the 4 point function alone. Mia's advice, don't be a square seems to pay off.

We would like to especially thank Jacob Abajian for collaboration at the initial stages of this project. We thank F. Alday, A. Homrich, and G. Korchemsky for comments on the draft. Research at the Perimeter Institute is supported in part by the Government of Canada through NSERC and by the Province of Ontario through MRI. This work was additionally supported by a grant from the Simons Foundation (PV: No. 488661). The work of V. G. was supported by the Coordenacao de Aperfeicoamento de Pessoal de Nivel Superior-Brasil (CAPES) and the Simons Foundation Grant No. 488637 (Simons Collaboration on the Non-perturbative bootstrap). Centro de Fsica do Porto is partially funded by Fundao para a Cincia e a Tecnologia (FCT) under the Grant No. UID04650-FCUP. The work of C. B was supported by Process $n^{\circ}$ 2018/25180-5, Fundação de Amparo à Pesquisa do Estado de São Paulo (FAPESP).

[1] D. Poland, S. Rychkov, and A. Vichi, The conformal bootstrap: Theory, numerical techniques, and applications, Rev. Mod. Phys. 91, 015002 (2019).

[2] L. F. Alday and A. Bissi, Higher-spin correlators, J. High Energy Phys. 10 (2013) 202.

[3] The superscript of $\mathcal{C}$ can be filled (black filled circle) or unfilled (white filled circle), associated with protected and unprotected operators, respectively.

[4] G. P. Korchemsky, Asymptotics of the Altarelli-ParisiLipatov evolution kernels of parton distributions, Mod. Phys. Lett. A 04, 1257 (1989); G. P. Korchemsky and G. Marchesini, Structure function for large $\mathrm{x}$ and renormalization of Wilson loop, Nucl. Phys. B406, 225 (1993); A. V. Belitsky, A. S. Gorsky, and G. P. Korchemsky, Logarithmic scaling in gauge/string correspondence, Nucl. Phys. B748, 24 (2006).

[5] L. F. Alday and J. M. Maldacena, Gluon scattering amplitudes at strong coupling, J. High Energy Phys. 06 (2007) 064.

[6] J. M. Drummond, G. P. Korchemsky, and E. Sokatchev, Conformal properties of four-gluon planar amplitudes and Wilson loops, Nucl. Phys. B795, 385 (2008).

[7] A. Brandhuber, P. Heslop, and G. Travaglini, MHV amplitudes in $N=4$ super Yang-Mills and Wilson loops, Nucl. Phys. B794, 231 (2008).

[8] J. M. Drummond, J. Henn, G. P. Korchemsky, and E. Sokatchev, On planar gluon amplitudes/Wilson loops duality, Nucl. Phys. B795, 52 (2008).
[9] J. M. Drummond, J. Henn, G. P. Korchemsky, and E. Sokatchev, Conformal Ward identities for Wilson loops and a test of the duality with gluon amplitudes, Nucl. Phys. B826, 337 (2010).

[10] L. F. Alday, D. Gaiotto, J. Maldacena, A. Sever, and P. Vieira, An operator product expansion for polygonal null Wilson loops, J. High Energy Phys. 04 (2011) 088.

[11] B. Basso, S. Komatsu, and P. Vieira, Structure constants and integrable bootstrap in planar $N=4$ SYM theory, arXiv:1505.06745; T. Fleury and S. Komatsu, Hexagonalization of correlation functions, J. High Energy Phys. 01 (2017) 130.

[12] B. Basso, A. Sever, and P. Vieira, Spacetime and Flux Tube $S$-Matrices at Finite Coupling for $N=4$ Supersymmetric Yang-Mills Theory, Phys. Rev. Lett. 111, 091602 (2013)

[13] S. Ferrara, A. F. Grillo, R. Gatto, and G. Parisi, Analyticity properties and asymptotic expansions of conformal covariant green's functions, Nuovo Cimento A 19, 667 (1974).

[14] M. S. Costa, J. Penedones, D. Poland, and S. Rychkov, Spinning conformal correlators, J. High Energy Phys. 11 (2011) 071.

[15] See Supplemental Material at http://link.aps.org/ supplemental/10.1103/PhysRevLett.126.121603 for a explicit construction of the conformal blocks and how cyclicity is imposed for the correlation functions, which includes Refs. [16,17].

[16] F. A. Dolan and H. Osborn, Conformal partial waves and the operator product expansion, Nucl. Phys. B678, 491 (2004).

[17] V. Goncalves, R. Pereira, and X. Zhou, 20' five-point function from $\mathrm{AdS}_{5} \times S^{5}$ supergravity, J. High Energy Phys. 10 (2019) 247.

[18] L. F. Alday, B. Eden, G. P. Korchemsky, J. Maldacena, and E. Sokatchev, From correlation functions to Wilson loops, J. High Energy Phys. 09 (2011) 123.

[19] When we take all $x_{i}$ and $x_{i+1}$ to be null separated we project into the $y$ polarization structure explicitly stripped out in Eq. (11) here so $G_{n}$ really only depends on the space-time cross ratios.

[20] We should identify the odd and even $v_{j}$ 's after taking the derivatives.

[21] The replacement of the sums by integrals is justified since in the null polygon limit both the spins and polarizations are very large. The factor of $1 / 4=(1 / 2)^{2}$ accounts for the original sums being only over even spins.

[22] L. F. Alday and A. Bissi, Crossing symmetry and Higher spin towers, J. High Energy Phys. 12 (2017) 118.

[23] Equation (16) is even simpler than Eq. (1) in a sense it is given by a simple exponential, without any gamma function prefactor. Of course, when constructing the relevant $5 \mathrm{pt}$ function summand as in Eq. (14) get back two gamma functions thus restoring the same kind of complexity of the 4 pt case. The simplicity of these 3 pt functions with more than one spinning operator should be related to the absence of wrapping interactions in its hexagon description due to the effective large size of all bridges involved; see also Ref. [24]. The poles in those gamma functions were interpreted as a manifestation of level crossing [25]; it would be very interesting to investigate if they are present at finite $l_{i j}$. 
[24] B. Basso and D. L. Zhong, Three-point functions at strong coupling in the BMN limit, J. High Energy Phys. 04 (2020) 076.

[25] G. P. Korchemsky, On level crossing in conformal field theories, J. High Energy Phys. 03 (2016) 212.

[26] N. Drukker and J. Plefka, The Structure of $n$-point functions of chiral primary operators in $N=4$ super Yang-Mills at one-loop, J. High Energy Phys. 04 (2009) 001.

[27] M. S. Bianchi, On structure constants with two spinning twist-two operators, J. High Energy Phys. 04 (2019) 059.

[28] B. Eden, G. P. Korchemsky, and E. Sokatchev, From correlation functions to scattering amplitudes, J. High Energy Phys. 12 (2011) 002.

[29] B. Basso, L. J. Dixon, and G. Papathanasiou, The Origin of the Six-Gluon Amplitude in Planar $\mathcal{N}=4$ SYM, Phys. Rev. Lett. 124, 161603 (2020).

[30] The function $\Gamma_{\text {oct }}$ first showed up in the beautiful work by Frank Coronado [31] in the computation of 4 point functions of local operators of very large $R$ charge (see also Ref. [32]). This function, therefore, shows up in at least three places: Coronado's 4 point functions; in the origin limit of null polygonal Wilson loops [29]; and governing the large spin and large polarization limit of spinning structure constants. Is this Letter we related the last two but the connection of either of them with Ref. [31] remains mysterious. It would be very interesting to understand this triangle of relations in more detail.

[31] F. Coronado, Bootstrapping the Simplest Correlator in Planar $\mathcal{N}=4$ Supersymmetric Yang-Mills Theory to All Loops, Phys. Rev. Lett. 124, 171601 (2020).

[32] A. V. Belitsky and G. P. Korchemsky, Octagon at finite coupling, J. High Energy Phys. 07 (2020) 219.
[33] The absence of any free parameters is expected. In the null limit the $5 \mathrm{pt}$ functions are related to pentagon Wilson loops, which are very simple in a conformal gauge theory since we cannot build cross ratios out of a null pentagon's insertions. This is why the BDS ansatz for (the dual) gluon scattering amplitudes [34] in planar $\mathcal{N}=4 \mathrm{SYM}$ is exact for five gluons [9].

[34] Z. Bern, L. J. Dixon, and V. A. Smirnov, Iteration of planar amplitudes in maximally supersymmetric Yang-Mills theory at three loops and beyond, Phys. Rev. D 72, 085001 (2005).

[35] J. F. Fortin, W. Ma, and W. Skiba, Higher-point conformal blocks in the comb channel, J. High Energy Phys. 07 (2020) 213.

[36] V. Rosenhaus, Multipoint conformal blocks in the comb channel, J. High Energy Phys. 02 (2019) 142.

[37] S. Parikh, A multipoint conformal block chain in $d$ dimensions, J. High Energy Phys. 05 (2020) 120.

[38] B. Basso, A. Sever, and P. Vieira, Space-time S-matrix and flux tube S-matrix II. Extracting and matching data, J. High Energy Phys. 01 (2014) 008.

[39] S. Caron-Huot, L. J. Dixon, J. M. Drummond, F. Dulat, J. Foster, Ö. Gürdoğan, M. von Hippel, A. J. McLeod, and G. Papathanasiou, The steinmann cluster bootstrap for $N=4$ super Yang-Mills amplitudes, Proc. Sci., CORFU2019 (2020) 003 [arXiv:2005.06735].

[40] A. L. Fitzpatrick, J. Kaplan, D. Poland, and D. SimmonsDuffin, The analytic bootstrap and AdS superhorizon locality, J. High Energy Phys. 12 (2013) 004.

[41] Z. Komargodski and A. Zhiboedov, Convexity and liberation at large spin, J. High Energy Phys. 11 (2013) 140. 